\title{
OPEN Electrophysiological characterization of a diverse group of sugar transporters from Trichoderma reesei
}

\author{
Sami Havukainen ${ }^{1}$, Jonai Pujol-Giménez ${ }^{2}$, Mari Valkonen ${ }^{1}$, Ann Westerholm-Parvinen ${ }^{1}$, \\ Matthias A. Hediger ${ }^{2}$ \& Christopher P. Landowski ${ }^{1 \bowtie}$
}

Trichoderma reesei is an ascomycete fungus known for its capability to secrete high amounts of extracellular cellulose- and hemicellulose-degrading enzymes. These enzymes are utilized in the production of second-generation biofuels and $T$. reesei is a well-established host for their production. Although this species has gained considerable interest in the scientific literature, the sugar transportome of $T$. reesei remains poorly characterized. Better understanding of the proteins involved in the transport of different sugars could be utilized for engineering better enzyme production strains. In this study we aimed to shed light on this matter by characterizing multiple $T$. reesei transporters capable of transporting various types of sugars. We used phylogenetics to select transporters for expression in Xenopus laevis oocytes to screen for transport activities. Of the 18 tested transporters, 8 were found to be functional in oocytes. 10 transporters in total were investigated in oocytes and in yeast, and for 3 of them no transport function had been described in literature. This comprehensive analysis provides a large body of new knowledge about $T$. reesei sugar transporters, and further establishes $X$. laevis oocytes as a valuable tool for studying fungal sugar transporters.

Lignocellulose biomass has gained interest as a feedstock for second generation biofuels due to its high abundance and renewability. It is used as a nutrition source by saprophytic fungi, which degrade its polysaccharide components into a range of soluble sugars. Trichoderma reese $i$ is a well-known species of saprophytic fungi that is used in the enzyme industry for the production of cellulose- and hemicellulose degrading enzymes (cellulases and hemicellulases), and which has been also studied as a platform for heterologous protein production. Although T. reesei has been the subject of numerous publications since its identification in the 1950s, sugar transporters coded in its genome remain poorly characterized.

Sugar transporters are membrane proteins which translocate sugars across the cell membrane. Fungal sugar transporters belong mainly to the major facilitator superfamily (MFS) ${ }^{1}$, whose members are characterized by the 12 transmembrane domain (TMD) topology². Few 7 TMD SWEETs (Sugars Will Eventually be Exported Transporter) have also been identified from fungi, but they are absent from phylum Ascomycota which contains industrially important fungi such as T. reesei and S. cerevisiae ${ }^{3}$. The MFS contains both passive facilitators and sugar $/ \mathrm{H}^{+}$symporters. Passive facilitators transport sugars along their concentration gradient across the cell membrane, while symporters are able to accumulate sugars against their concentration gradient by coupling the transport process to the transport of a co-substrate which follows its concentration gradient. In the known fungal sugar symporters the co-substrates are protons, in contrast to sodium ions used by many sugar symporters from animals, that belong to a different family of transporters ${ }^{4}$.

The yeast Saccharomyces cerevisiae is the most studied species of fungi, and relevant to biofuel processes as the organism responsible for fermenting the lignocellulose-derived sugars to ethanol. Its well-characterized sugar transport system relies solely on passive facilitators in the transport of hexose sugars, which are present at high concentrations in its natural habitat ${ }^{5}$. On the other hand, the natural habitat of saprophytic fungi contains wide variety of biomass-derived sugars which are available only at low concentrations ${ }^{6}$. This wider range of utilizable substrates is reflected by the high number of sugar transporter genes when compared to S. cerevisiae ${ }^{7}$. The scarcity of sugars in the natural habitat is reflected by the fact that fungal high-affinity monosaccharide transporters often

${ }^{1}$ Protein Production Team, VTT Technical Research Center of Finland Ltd, Tietotie 2, 02150 Espoo, Finland. ${ }^{2}$ Membrane Transport Discovery Lab, Department of Biomedical Research, Inselspital, University of Bern, 3010 Bern, Switzerland. ${ }^{\square}$ email: christopher.landowski@vtt.fi 
have their affinities in the micromolar range $\mathrm{e}^{7-11}$, whereas the affinities of their S. cerevisiae counterparts are in the millimolar range ${ }^{12}$. Additionally, many fungal monosaccharide transporters appear to utilize the active sugar/ $\mathrm{H}^{+}$ symport mechanism, again unlike $S$. cerevisiae, which further suggests adaptation to low sugar environments ${ }^{10}$. Fungal sugar transporters have been proven useful for engineering S. cerevisiae to uptake biomass-derived sugars (e.g. D-xylose, L-arabinose and D-galacturonic acid) whose transport is not otherwise optimal in yeast, and whose utilization is required for an economically competitive bioethanol production process ${ }^{13-15}$.

Although the genome of $T$. reesei has been predicted to contain about 50-100 genes coding for sugar transporters ${ }^{1,7,16}$, only handful of them have been characterized in the literature (Reviewed in Ref. ${ }^{1}$ ). Transporters have thus far been identified for monosaccharides, $\beta$-linked disaccharides and sugar acids ${ }^{7,14,17-21}$. Several transporter genes have been observed to be upregulated when the fungus is grown on cellulase-inducing carbon sources, and to be regulated by transcription factors involved in cellulase induction ${ }^{22-25}$. As different sugars act as inducers or repressors of the cellulolytic system and since these sugars are believed to be sensed intracellularly, sugar transporters play an important role in cellulase induction in saprophytic fungi ${ }^{26,27}$. Indeed, the deletion of sugar transporter genes has been shown to affect cellulase production, sugar transport and growth in $T$. reesei $i^{6,17,19,23,28}$.

Inducers of the cellulase and hemicellulase gene expression in T. reesei include cellobiose, sophorose, lactose and $\mathrm{L}$-sorbose $\mathrm{e}^{26}$. Of these, lactose is relevant to industrial applications due to its low price and solubility ${ }^{23}$. D-Glucose, which is easily metabolized, represses the production of cellulases and hemicellulases via phenomenon termed carbon catabolite repression (CCR ${ }^{29}$. The preference for D-glucose is illustrated by the fact that many fungal species possess multiple glucose transporters with varying affinities, capacities and expression patterns $s^{8,12}$. The tight regulation of cellulase induction by different sugars could possibly enable the improvement of cellulase production via manipulation of sugar transporters. This would be desirable, as these enzymes form a significant fraction of the manufacturing cost of second generation bioethanol ${ }^{30}$. Examples would be the alleviation of CCR by deletion of D-glucose transporters, or the improvement of inducer uptake by overexpression of e.g. lactose transporters. The prerequisite for both of these approaches is the knowledge of the sugar transportome of the species in question.

Fungal sugar transporters have been characterized mainly by heterologous expression in S. cerevisiae with transport experiments carried out with radiolabeled sugars. Transporters from other kingdoms of life have been characterized with electrophysiological methods (Reviewed for plant transporters in Ref. ${ }^{31}$ ), such as two-voltage electrode clamp (TEVC) $)^{32}$, but to our knowledge there are only two published studies where this method has been applied to fungal sugar transporters ${ }^{33,34}$. In this method, the transporter gene is expressed in Xenopus laevis oocytes and the flow of ions through their plasma membrane is monitored ${ }^{32}$. To do so, the cell membrane is clamped to a specific voltage (analogous to physiological resting membrane potential) and the current required to keep this voltage constant is recorded. Electrophysiological studies are convenient for transporter functional characterization, since they do not require expensive and limitedly available radiolabeled sugars, and since a complete set of kinetics can be measured on a single oocyte simultaneously with multiple test voltages ${ }^{35}$. Nevertheless, since these methods measure the flow of ions into the cell, they are limited to the characterization of electrogenic transporters, e.g. sugar $/ \mathrm{H}^{+}$symporters. In this regard, many fungal sugar transporters appear to function as symporters, and thus they could be studied with this method.

Accordingly, we set to investigate $T$. reesei transporters with electrophysiological methods. Our aim was to gain better understanding of T. reesei sugar transporters, and to further establish the TEVC method for the analysis of fungal sugar transporters. We used phylogenetic analysis to identify a convenient set of transporters for the functional studies, with the aim of selecting transporters for many different classes of sugars. Some of these transporters had been published before, but our analysis provided new information about their substrates and kinetics. We also identified and characterized some transporters for which no transport activity had been described before.

\section{Results and discussion}

Selection of $T$. reesei sugar transporters for functional studies. Phylogenetic analysis was used to identify interesting transporters for the functional studies. Putative T. reesei sugar transporters were chosen for sequence alignment with previously characterized fungal sugar transporters and a phylogenetic tree was built from the alignment (Fig. 1). We further annotated the tree with substrates known to be transported by the already characterized transporters, which allowed us to assign distinct clades for different substrates. Clades were seen for example for $\beta$-linked disaccharide transporters (Fig. 1a, middle part) and sugar acid transporters (Fig. 1b, middle part). A less defined clade containing $\alpha$-linked disaccharide transporters was also visible (Fig. 1a, upper part). Monosaccharide transporters were the most abundant type of transporter in the tree (Fig. 1a lower part, b upper part), which is not surprising since D-glucose is the preferred carbon source for $T$. reesei. Some clades of monosaccharide transporters seemed to be enriched in their ability to transport pentose sugars (Fig. 1b, lower part).

We selected 16 transporters for further analysis (Table 1). These transporters represented different clades of the phylogenetic tree shown in Fig. 1. Although several of these transporters had been published before, we hypothesized that electrophysiological analysis could provide new insights into their function. To complement the phylogenetic analysis, we surveyed published literature about regulation of gene expression by different carbon sources $^{23,36}$, or by the major transcription factors linked to cellulase expression (XYR1, ACE3) or carbon catabolite repression (CRE1) $)^{24,25,29}$. Many of the selected transporters had been identified to be significantly upregulated upon growth on inducing carbon sources, cellulose and lactose (Table 1). Interestingly, Trire2_106556 showed the opposite pattern as it was downregulated when the fungus was grown on these carbon sources. Many transporters were downregulated in strains lacking either of the cellulase regulators XYR1 or ACE ${ }^{24,37}$, with CRT1 $1^{19,21,23}$ 


\begin{tabular}{|c|c|c|c|c|c|c|c|c|c|c|}
\hline \multirow[b]{2}{*}{ Trire2 } & \multirow[b]{2}{*}{ Clade } & \multicolumn{2}{|c|}{ C-source } & \multicolumn{3}{|c|}{ Transcription factor } & \multirow[b]{2}{*}{$\mathbf{H}^{+}$} & \multicolumn{2}{|c|}{ Substrates } & \multirow[b]{2}{*}{ Refs. } \\
\hline & & $\mathrm{Cel} / \mathrm{Lac}$ & Lac & XYR1 & ACE3 & CRE1 & & New & Known & \\
\hline 50618 & Mono/c6 & $\uparrow$ & & & & $\uparrow$ & $\mathrm{x}$ & fru & & \\
\hline 72383 & Mono/c6 & & & & & & & & & \\
\hline $79202^{\mathrm{a}}$ & Mono/c6 & $\uparrow$ & $\uparrow$ & & $\downarrow$ & $\uparrow$ & & & & \\
\hline 106556 & Mono/c6 & $\downarrow$ & $\downarrow$ & & & $\downarrow$ & & & & \\
\hline STR3 $^{\mathrm{a}}$ & Mono/c6 & & & & & & $\mathrm{x}$ & sor & glc, fru, gal, mann, xyl & 7 \\
\hline 62502 & Mono/c5 & $\uparrow$ & & & & & & & & \\
\hline STR1 & Mono/c5 & $\uparrow$ & $\uparrow$ & $\downarrow$ & & & $\mathrm{x}$ & ara & glc, fru, gal, mann, xyl & 7,17 \\
\hline $56684^{\mathrm{a}}$ & $\mathrm{Di} / \beta$ & $\uparrow$ & $\uparrow$ & & $\downarrow$ & & & & & \\
\hline $67752^{\mathrm{a}}$ & $\mathrm{Di} / \beta$ & $\uparrow$ & $\uparrow$ & & & & & & $\mathrm{cb}$ & 20 \\
\hline 77517 & $\mathrm{Di} / \beta$ & $\uparrow$ & & & & & & & & \\
\hline $\mathrm{CRT1}^{\mathrm{a}}$ & $\mathrm{Di} / \beta$ & $\uparrow$ & $\uparrow$ & $\downarrow$ & $\downarrow$ & $\uparrow$ & $\mathrm{x}$ & glc, sop ${ }^{b}$ & $\mathrm{cb}, \mathrm{lac}$ & 21 \\
\hline 65191 & $\mathrm{Di} / \alpha$ & $\uparrow$ & & $\downarrow$ & & & & & & \\
\hline $67469^{\mathrm{a}}$ & $\mathrm{Di} / \alpha$ & & $\uparrow$ & & & $\uparrow$ & $\mathrm{x}$ & glc & & \\
\hline 69957 & $\mathrm{Di} / \alpha$ & $\uparrow$ & $\uparrow$ & $\downarrow$ & $\downarrow$ & & & & mann, xyl, cb & 38 \\
\hline 69026 & Acid & $\uparrow$ & $\uparrow$ & & & & $\mathrm{x}$ & glcUA & galUA & 14 \\
\hline 106330 & Acid & $\uparrow$ & & & & & $\mathrm{x}$ & glcUA & galUA & 14 \\
\hline
\end{tabular}

Table 1. T. reesei transporters tested in this study for symport activity in oocytes and their regulation by carbon sources and transcription factors. Clade refers to clades identified from Fig. 1. Significantly differential expression (DE) by carbon sources or by transcription factors was inferred from published studies ${ }^{23-25,29,36}$. $\mathrm{Cel} / \mathrm{Lac}=\mathrm{DE}$ on cellulose or lactose versus $\mathrm{D}$-glucose $\mathrm{c}^{36}, \mathrm{Lac}=\mathrm{DE}$ on lactose versus glycerol or $\mathrm{D}$-glucose $\mathrm{g}^{23}$, XYR1 $=$ DE on cellulose or $\alpha$-sophorose between $\triangle x y r 1$ and parental QM9414 strains ${ }^{24}$, ACE3 = DE on lactose between $\triangle a c e 3$ and parental QM6a strains ${ }^{25}$, CRE1 = DE on D-glucose between $\Delta c r e 1$ and parental QM9414 strains ${ }^{29} . \mathrm{H}^{+}$indicates symport activity detected in this study. Abbreviations as in Fig. 1. ${ }^{\text {a Sequence differs from }}$ that listed in the QM6a genome assembly (See Materials and methods). ${ }^{\mathrm{b}} \alpha$-sophorose.

and Trire2_69957 $7^{38}$ being regulated by both. Several transporters were also upregulated on D-glucose in the absence of carbon catabolite repression regulator CRE1, which suggests that these transporters participate in the transport of sugars other than D-glucose.

The selected transporters were cloned into a $X$. laevis expression vector, and mRNA generated from these constructs was injected into X. laevis oocytes. In addition to the transporters listed in Table 1, we included Neurospora crassa cellodextrin transporters CDT-1 and CDT-2 as positive and negative controls, respectively. CDT- 1 has been shown to function via symport mechanism and CDT-2 as a facilitator ${ }^{39}$, and as previously mentioned, TEVC can only be used for transporters which move net charges, such as symporters. The oocytes were screened for transport activity by perfusing them with different sugars while the electrical current generated across the cell membrane was measured continuously. Each transporter was tested with at least 11 different sugars, including hexose and pentose monosaccharides, as well as $\beta$ - and $\alpha$-linked disaccharides (see Materials and Methods). Some transporters were also tested with additional sugars or sugar acids on the basis of information from the phylogenetic tree (e.g. Trire2_69026 and_106330 with uronic acids).

Of the 16 T. reesei and $2 N$. crassa transporters tested, 8 proved out to be functional in oocytes (Table 1 ), as sugar-induced currents were seen in oocytes expressing these transporters (Supplementary Figure S1). No sugar-induced currents were seen in oocytes expressing the other transporters, or in those injected with water (data not shown). The direction of current was inwards, indicating flow of positive ions into the cell, which we hypothesized to be caused by sugar $/ \mathrm{H}^{+}$symport activity. Of the 8 identified symporters, transport function had been previously demonstrated for 6 (Table 1$)$, and $2\left(\right.$ CDT- $1^{39}$ and CRT $1^{21}$ ) were known to be symporters.

We did further tests in yeast with the transporters that did not have symport activity in oocytes and which had not been functionally characterized in the literature. Yeast strains expressing these transporters were tested for D-glucose uptake, as shown in Supplementary Figure S2. With this further screening we were able to identify one additional transporter, Trire2_106556, whose expression enabled yeast to uptake D-glucose and grow on multiple hexose sugars (Supplementary Figure S2). After identifying a set of functional transporters, we did some more specific experiments to characterize them.

Multiple proteins are responsible for D-glucose transport in T. reesei. Our analysis identified the previously published $\mathrm{D}$-glucose/D-xylose transporters STR $1^{7,17}$ and STR3 ${ }^{7}$ to function via symport mechanism. We further studied their substrate selectivity by recording currents induced by different sugars across multiple test voltages. Representative examples of these I-V curves are shown in Supplementary Figure S3 and the currents obtained at $-50 \mathrm{mV}$ test voltage are shown in Fig. 2a,b. Both transporters proved out to be able to transport multiple monosaccharide sugars, which is in line with previously published results ${ }^{7}$. In addition to previously reported substrates, we identified STR1 to be able to transport L-arabinose and STR3 to be able to transport L-sorbose, which both have been identified as hemicellulase or cellulase inducers in T. reesei, respectively ${ }^{40-42}$. 


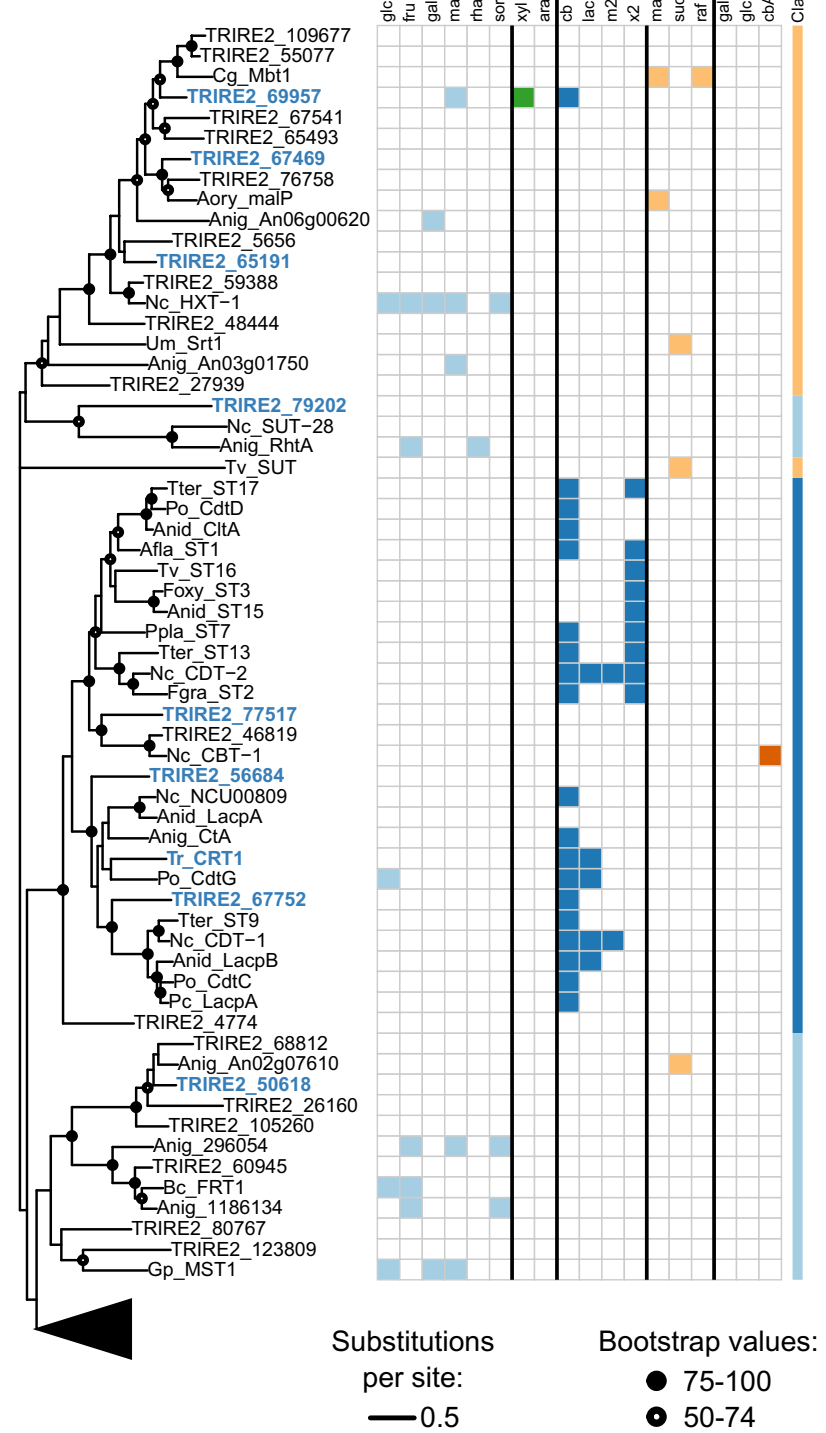

b

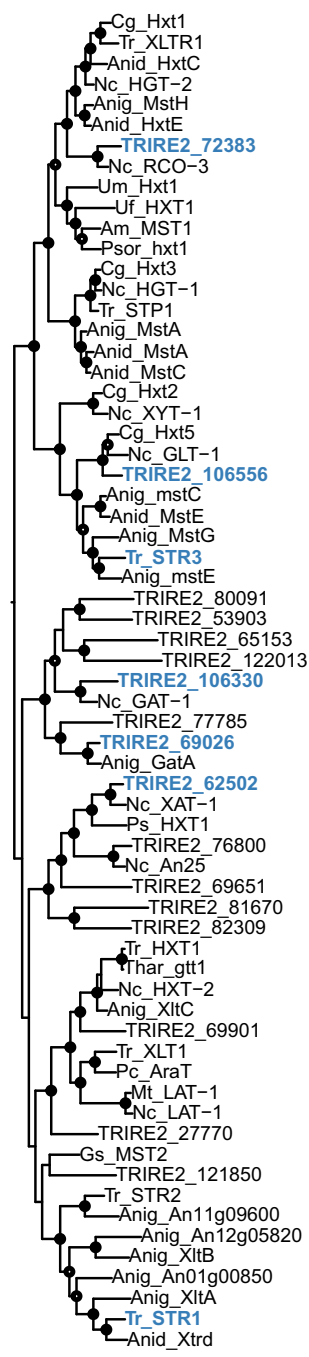

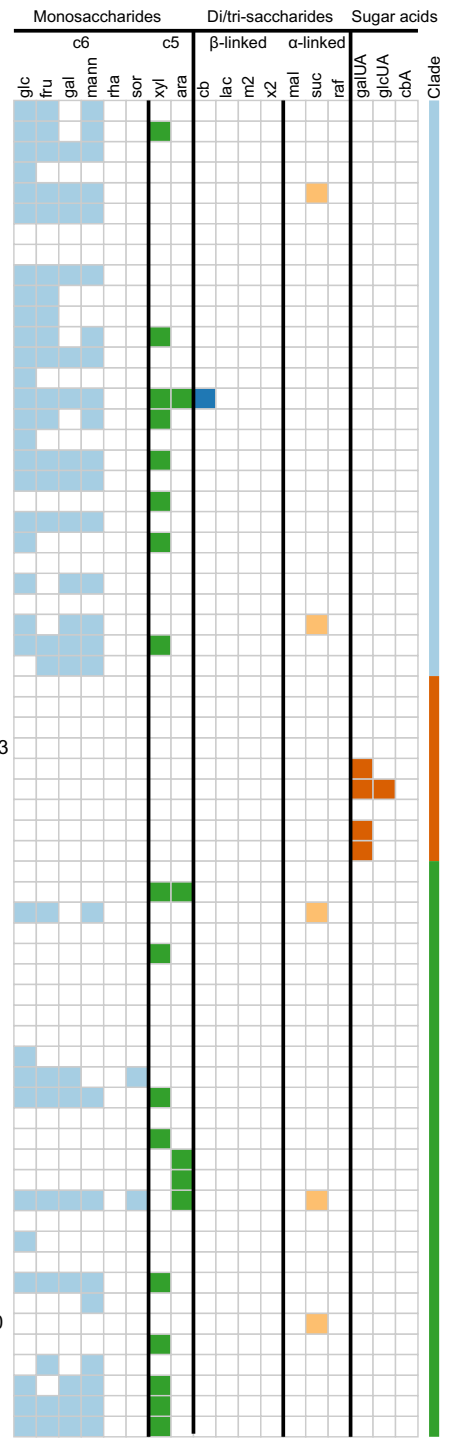

Figure 1. Phylogenetic tree of published sugar transporters from filamentous fungi and putative T. reesei sugar transporters. (a, b) Phylogenetic tree built from sequence alignment of published fungal sugar transporters and putative T. reesei transporters. (b) Presents the expansion of the hidden clade from (a). Known substrates of the transporters are indicated (References and organisms are listed in Supplementary Table S1). Substrates are divided into monosaccharides (c6: hexoses, c5: pentoses), disaccharides ( $\beta$ - and $\alpha$-linked) and sugar acids. $T$. reesei transporters which were selected for expression in oocytes are indicated in bold and with color. Scale bar presents substitutions per site and the symbols present bootstrap support values from 100 runs. Abbreviations: glc $=\mathrm{D}$-glucose, fru $=\mathrm{D}$-fructose, gal $=\mathrm{D}$-galactose, mann $=\mathrm{D}$-mannose. rha $=\mathrm{L}$-rhamnose, sor $=\mathrm{L}$-sorbose, $\mathrm{xyl}$ $=\mathrm{D}$-xylose, ara $=\mathrm{L}$-arabinose, $\mathrm{cb}=$ cellobiose, lac $=$ lactose, $\mathrm{m} 2=$ mannobiose, $\mathrm{x} 2=$ xylobiose, $\mathrm{mal}=$ maltose, suc $=$ sucrose, raf $=$ raffinose, galUA $=$ D-galacturonic acid, glcUA $=$ D-glucuronic acid, $c b A=$ cellobionic acid . The figure was created with ape (version 5.4-1) package for $\mathrm{R}$ (version 4.0.3) ${ }^{79,84}$.

We also analyzed transport kinetics for the main substrates of these transporters (Fig. 2b,c). STR1 had high affinity for both D-glucose and D-xylose, but low affinity for L-arabinose. STR3 had high affinity for D-glucose and $\mathrm{D}$-mannose, but lower affinities for D-galactose and D-xylose. The kinetics for D-glucose and D-xylose have been determined previously in yeast ${ }^{7}$, and our results are mostly in agreement with them (see below). With the oocyte system we could analyze the kinetics at multiple test voltages (Supplementary Figure S4). With this analysis we discovered that the affinity of both transporters for their substrates decreased as the voltage became more depolarized, except with D-glucose for which clear trends were not seen (Supplementary Figure S5). Transport by STR1 was also found to be more voltage-dependent than that of STR3, as judged by the larger increase in maximum transport rate across the test voltages (Supplementary Figure S6).

One of the transporters identified from the phylogenetic analysis and expression data survey was Trire2_106556, which was here named GLT1 (glucose transporter 1). It clustered together with low-affinity 

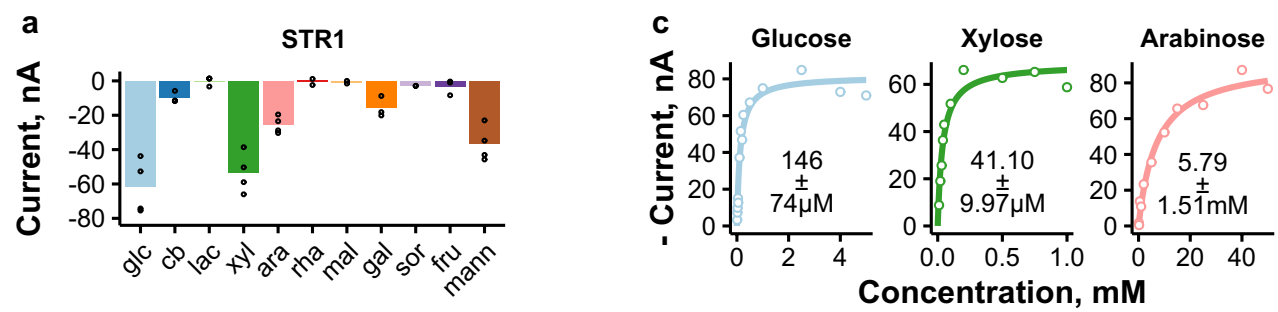

\section{Substrate}
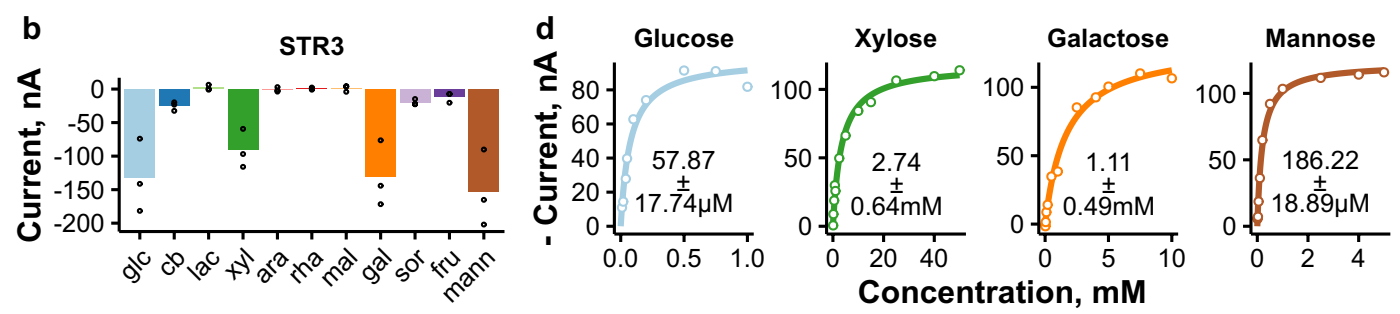

Substrate

Figure 2. Electrophysiological characterization of D-glucose transporters STR1 and STR3. (a, b) Selectivity plots of STR1 (a) and STR3 (b) measured by recording currents in the presence of different sugars in $5 \mathrm{mM}$ concentration (abbreviations as in Fig. 1). Results obtained with $-50 \mathrm{mV}$ test voltage are shown. Points present results obtained from individual oocytes and bars their mean $(n \geq 3)$. (c, d) Sugar transport kinetics of STR1 (c) and STR3 (d) at $-50 \mathrm{mV}$. Representative experiments are shown. Lines present the Michaelis Menten kinetics prediction and the $K_{\mathrm{m}}$ values present the mean and standard deviation from individual oocytes ( $n=3$, except $n=5$ for D-glucose kinetics of STR3). The figure was created with ggplot2 package (version 3.3.2) for $\mathrm{R}^{79,85}$.

D-glucose transporters from N. crassa and C. graminicola 8,11 (Fig. 1), and, in contrast to the majority of the selected transporters, it was upregulated on D-glucose (Table 1 and Ref. ${ }^{43}$ ). Although both yeast (Supplementary Figure S2) and oocytes (data not shown) expressing this transporter were able to uptake D-glucose, no sugarinduced currents were detected with TEVC from oocytes expressing this transporter. Therefore we hypothesized that it functions as a passive facilitator. Analysis of the transport kinetics in yeast proved that GLT1 is a lowaffinity D-glucose transporter (Fig. 3a). The results contradict a recent study where no transport activity was detected in yeast strain expressing GLT $1{ }^{18}$. However, since the used amino acid sequences were identical, we can't offer a reason for this discrepancy. In the same conditions, a linear relationship between the uptake rate and D-glucose concentration was observed in the negative control strain (Fig. 3a), which is consistent with earlier reports $^{12}$. The used strain was based on the EBY.VW5000 background, in which some low-affinity D-glucose uptake is observed due to the SNF3 deletion ${ }^{44}$.

The function of GLT1 is in line with its homology to low-affinity D-glucose transporter GLT-1 from N. crassa (Fig. 1). The D-glucose transport system of N. crassa also includes two high-affinity transporters (HGT-1 and $-2)^{8}$. Their T. reesei homologs are facilitators STP $1^{19,45,46}$ and XLTR $1^{18}$ (Fig. 1), of which the latter appears to be expressed only at low levels based on published transcriptome data sets ${ }^{22,23}$. On the other hand, STP1 has been found to be expressed constitutively across multiple carbon sources (Table S1 of Ref. ${ }^{36}$ ). We expressed STP1 in yeast to study its D-glucose transport kinetics, since as a passive facilitator ${ }^{46}$ it could not be studied with TEVC. As hypothesized from the phylogenetic analysis, STP1 proved out to be high-affinity D-glucose transporter (Fig. 3b).

We also did competition experiments for GLT1, STR1 and STR3 with the yeast system to further investigate their substrate specificity. D-glucose transport by GLT1 was not inhibited significantly by any of the tested sugars in 5-fold excess except by D-glucose itself (as expected) and slightly by D-mannose (Fig. 3c), suggesting that D-glucose is the primary substrate. Regarding STR1, its D-glucose transport activity was heavily inhibited by D-xylose (Fig. 3d), which is in line with its high affinity for this substrate (Fig. 2c). D-glucose transport by STR3 was inhibited by D-glucose, D-mannose and D-galactose (Fig. 3e), as expected from the kinetics results (Fig. 2d). We did not include STP1 in the competition experiments, since a similar experiment had been done previously $^{46}$. In the previous study, the transport of a fluorescent D-glucose analog was found to be inhibited severely by $\mathrm{D}$-glucose, $\mathrm{D}$-mannose and $\mathrm{D}$-fructose, less severely by $\mathrm{D}$-xylose and only slightly by L-arabinose when the competing sugars were in 10 -fold excess ${ }^{46}$.

The discovery of low-affinity D-glucose transporter GLT1, identification of the symport mechanism and new substrates for STR1 and STR3 and the kinetic characterization of two important D-glucose transporters (STP1, GLT1) provide new information about the D-glucose transport system of T. reesei. Although our kinetics analysis for STR1 and STR3 was mostly in agreement with a previous study ${ }^{7}$, as shown in Supplementary Table S2, we noticed that only low affinity D-xylose transport activity had previously been described for STR1. However, we observed that the D-xylose kinetics from a previous report appeared to exhibit biphasic behavior, with highaffinity $(0-5 \mathrm{mM})$ and low affinity $(5-50 \mathrm{mM})$ components (Additional File 7 in Ref. ${ }^{7}$ ). Since the previously reported kinetics parameters were from a curve fitted to the whole concentration range, a separate fit for the high-affinity component would have resulted in a significantly lower $K_{\mathrm{m}}$ value. The high affinity for D-xylose was 

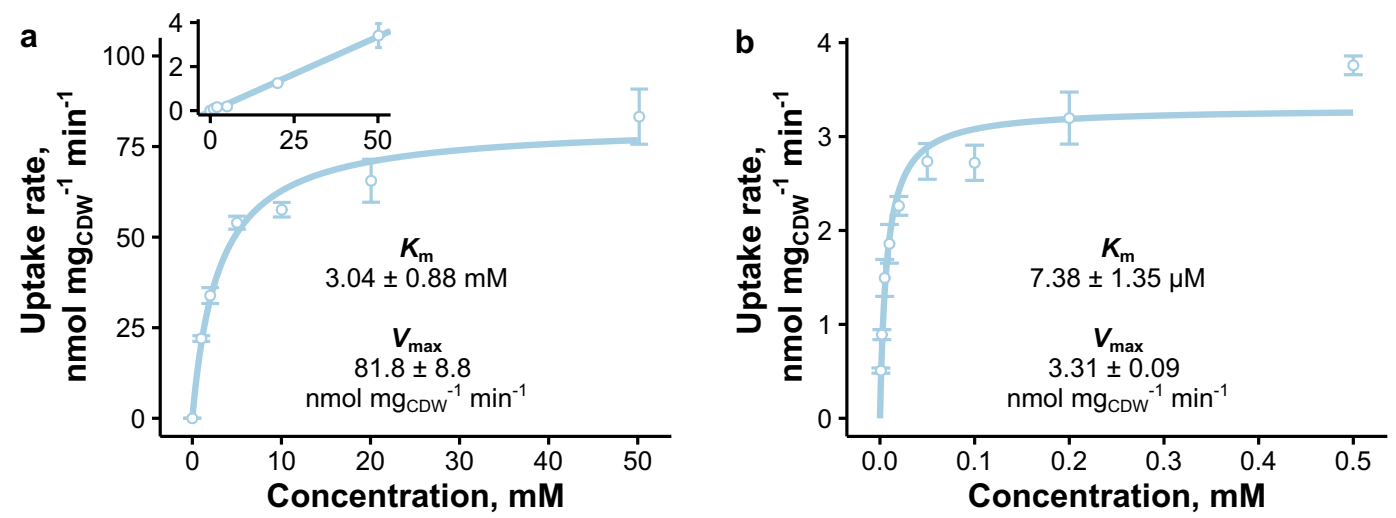

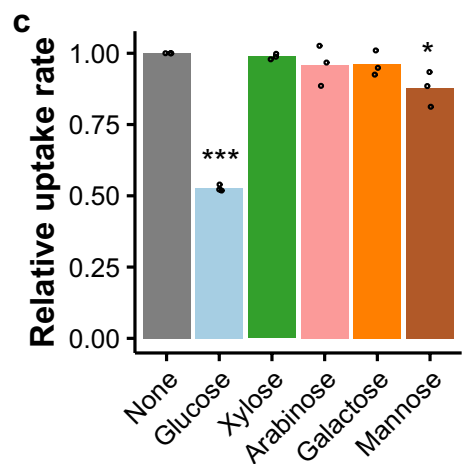

Inhibitor

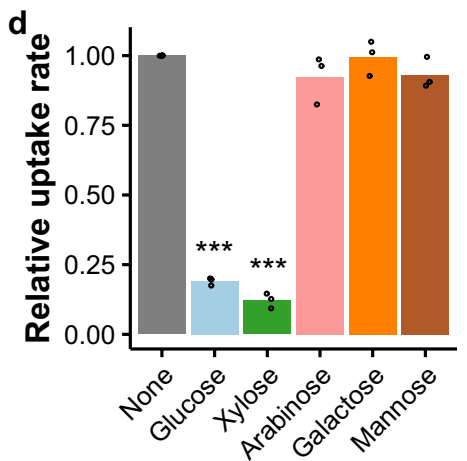

Inhibitor e

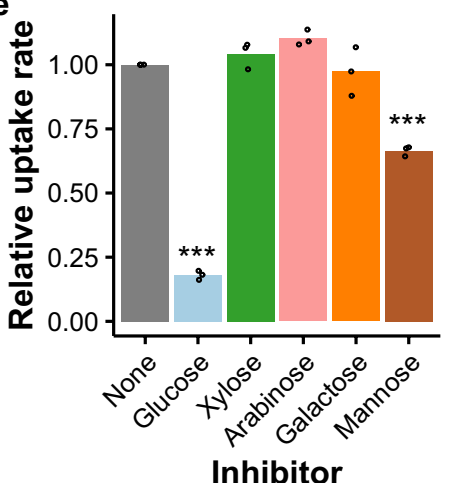

Figure 3. Further D-glucose transporter testing in yeast. (a, b) Glucose uptake kinetics of GLT1 (a) and STP1 (b) at $\mathrm{pH}$ 6.5. Points and error bars present mean uptake rates and standard deviation between biological replicates $(n=3$, except $n=2$ in the inset in a). Lines present the Michaelis-Menten kinetics prediction of the combined values from all biological replicates. The presented $K_{\mathrm{m}}$ and $V_{\max }$ values are the mean and standard deviation of kinetics parameters obtained from 3 biological replicates. Inset in a shows D-glucose uptake rate of the negative control strain with the line representing linear fit to the data. (c-e): Inhibition of glucose transport of GLT1 (c), STR1 (d) or STR3 (e) in a competition assay with inhibitors in 5-fold excess (1 vs $5 \mathrm{mM}$ ) at pH 6.5. Points present values obtained for biological replicates and bars their mean $(n=3)$. Significance in relation to condition without inhibitor was estimated with ANOVA and Tukey's honest significant difference test $\left({ }^{*}: p<\right.$ $\left.0.05,{ }^{* * *}: p<0.005\right)$. The figure was created with ggplot 2 package for $\mathrm{R}^{79,85}$.

further supported by the competition experiment, which showed that D-glucose transport by STR1 was heavily inhibited by D-xylose. The XYR1-dependent upregulation of STR1 in cellulase-inducing conditions (Table 1) as well as upon growth on D-xylose, and the severe growth defect of the str1 deletion strain on D-xylose and $\mathrm{L}$-arabinose, but not on $\mathrm{D}$-glucose, further support its physiological role as a pentose transporter rather than as a D-glucose transporter ${ }^{7,17}$. Indeed, the L-arabinose transport activity discovered here could explain the growth defect of $\Delta s t r 1$ strain on this sugar ${ }^{17}$.

Cellodextrin transporters CDT-1 and CRT1 have differences in substrate selectivity and in their affinity for protons. Our initial screening identified two previously published cellobiose symporters, CDT-1 and CRT1, to be functional in oocytes. Both are important for cellulase induction. In T. reesei, deletion of CRT1 is enough to abolish cellulase induction, whereas in N. crassa deletion of both CDT-1 and another cellobiose transporter CDT-2 is required ${ }^{19,23,47}$. Both CDT-1 and CRT1 have been shown to be able to transport cellobiose and lactose when expressed in yeast ${ }^{21,48}$, and this was also seen in oocytes (Fig. 4a,b). Kinetics measurements for these sugars, shown in Fig. 4c,d, revealed results following a similar trend as seen in previous studies, where the kinetics were determined in yeast (Supplementary Table S3). The results showed that cellobiose was transported with higher affinity compared to lactose and that CDT-1 had higher affinity for these two substrates compared to CRT1.

Regarding other substrates, CRT1 could also transport D-glucose, but its affinity for this sugar was about 100-200-fold lower than for cellobiose or lactose. D-Glucose transport has also been observed for its closest homolog in Fig. 1, P. oxalicum $\mathrm{CdtG}^{49}$. Additional experiments also indicated that CRT1 was able to transport the potent cellulase inducer $\alpha$-sophorose (Supplementary Figure S7). This observation could provide explanation for previous reports that have shown that CRT1 is indispensable for $\alpha$-sophorose mediated cellulase induction, although opposite findings have also been reported ${ }^{19,21,23}$. In one published study, CRT1 deletion did not appear to affect $\alpha$-sophorose uptake ${ }^{19}$, which indicates that there might be other transporters for $\alpha$-sophorose and thus 


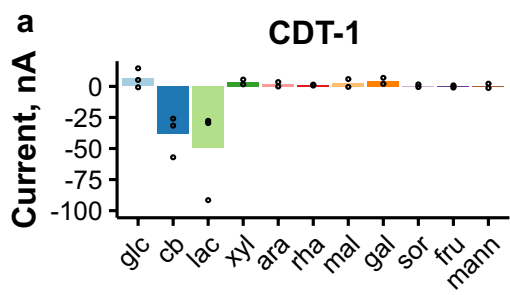

Substrate

C

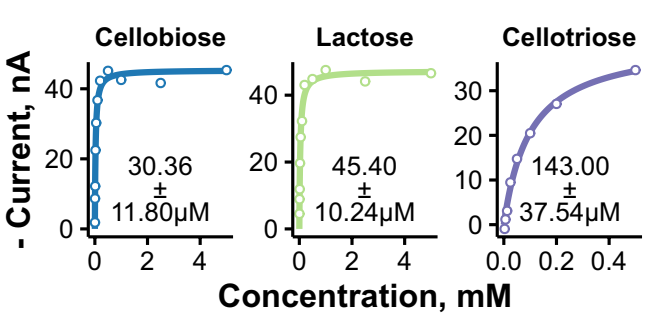

d

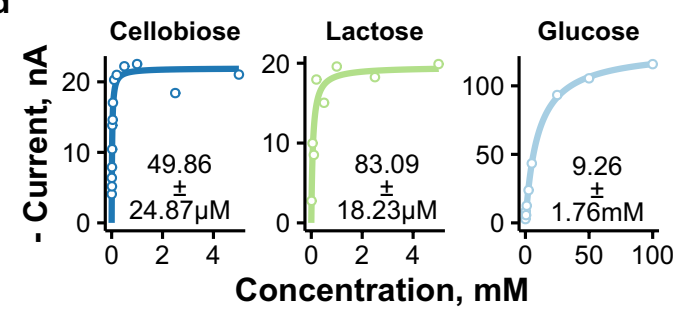

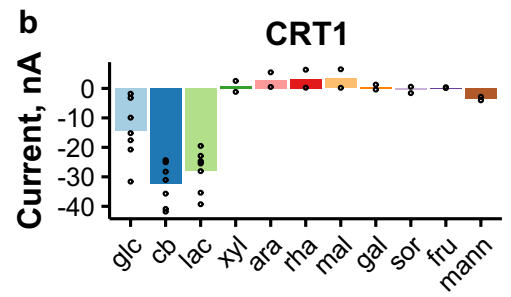

Substrate

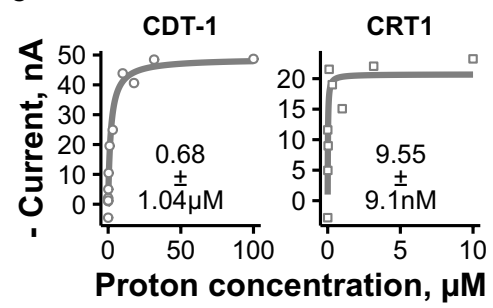

f

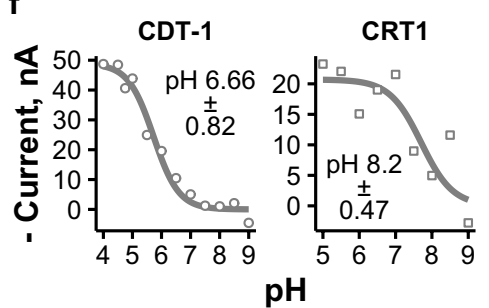

Figure 4. Electrophysiological characterization of cellodextrin transporters CDT-1 and CRT1. (a, b) Selectivity plots of CDT-1 (a) and CRT1 (b) measured by recording currents in the presence of different sugars in $5 \mathrm{mM}$ concentration (abbreviations as in Fig. 1). Results obtained with $-50 \mathrm{mV}$ test voltage are shown. Points present results obtained from individual oocytes and bars their mean $(n \geq 2)$. (c, d) Sugar transport kinetics of CDT-1 (c) and CRT1 (d) at - $50 \mathrm{mV}$. (e, f) Dependence of cellobiose transport by CDT-1 and CRT1 on proton concentration, with the proton concentration presented on linear (e) and log scale (f). Lines in panels (c-f) present the Michaelis Menten kinetics prediction, and the $K_{\mathrm{m}}$ values present mean and standard deviation from individual oocytes ( $n=3$, except $n=4$ for cellobiose kinetics of CRT1). Representative experiments are shown in panels (c-f). The figure was created with ggplot2 package for $\mathrm{R}^{79,85}$.

makes the understanding of components needed for induction more intriguing. $\alpha$-sophorose transport was not tested with CDT- 1 since $\alpha$-sophorose does not function as an inducer in N. crassa ${ }^{47}$.

Besides cellobiose and lactose, CDT-1 has been shown to be able to transport cellodextrins cellotriose and cellotetraose $\mathrm{e}^{48}$. We were interested in determining kinetics for these longer sugars, as this was not addressed in previous studies. Cellodextrins are probably rather abundant in the natural breakdown products of cellulose. Thus it is important to understand how short the enzyme-degraded sugars need to be in order to be taken up by the fungal cell, since the cellulose filaments are thousands of D-glucose units long. As expected based on previous reports, cellotriose was transported by oocytes expressing CDT-1 (Supplementary Figure S7). The affinity of CDT-1 for cellotriose was high, although lower than for cellobiose and lactose (Fig. 4c). We did not detect cellotetraose transport in oocytes, and thus we were unable to do the kinetic analysis for this sugar. Previously reported results from growth and inhibition tests have indicated that cellotetraose is transported, although less efficiently than cellotriose $\mathrm{e}^{21,48}$. Therefore, the cellotetraose concentration in our assay could have been too low to detect transport.

We also analyzed the $\mathrm{pH}$-dependence of these transporters by measuring the currents induced by $5 \mathrm{mM}$ cellobiose as a function of proton concentration (Fig. 4e). CRT1 had higher affinity for protons than CDT-1, and thus it is able to function in a broader $\mathrm{pH}$ range than CDT-1 (Fig. 4f). We are aware of a single fungal transporter whose $\mathrm{pH}$-dependence has been characterized before with TEVC ( $U$. maydis Srt1) ${ }^{33}$, and its $K_{\mathrm{m}}$ for protons is quite similar to that obtained for CRT1 (CRT1: $\mathrm{pH} 8.2 \pm 0.47$ vs Srt1: $\mathrm{pH} 7.7 \pm 0.31$ ). The observed differences in the affinity for protons are interesting, since both $N$. crassa and T. reesei are usually grown in slightly acidic conditions and both employ saprotrophic lifestyle. Regarding voltage kinetics, a slight increase in affinity was seen for CRT1 as the voltage became more depolarized (Supplementary Figure S8).

Identification of novel low-affinity hexose transporters Trire2_50618 (FRT1) and Trire2_67469 (MLT1). Two novel monosaccharide transporters, Trire2_50618 and_67469, were identified from the initial screening (Supplementary Figure S1) as being able to transport D-fructose and D-glucose, respectively. They were named FRT1 and MLT1, for fructose transporter 1 and maltose transporter-like protein 1, respectively. More 


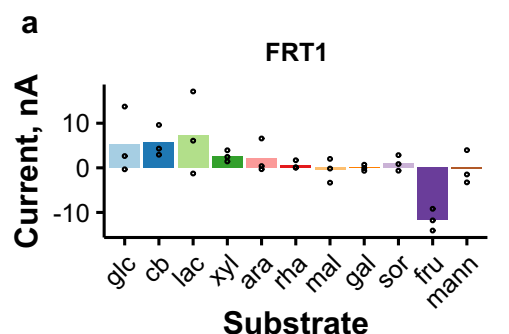

b

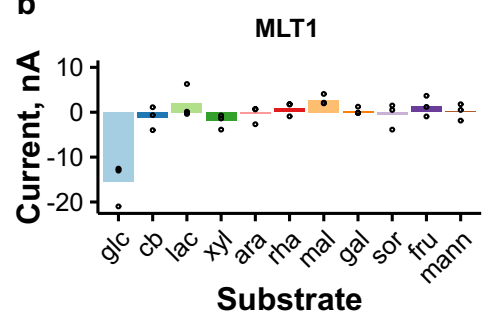

C

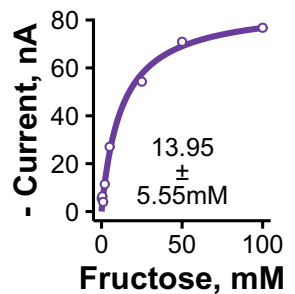

d

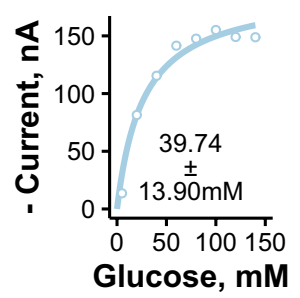

Figure 5. Electrophysiological characterization of hexose transporters FRT1 and MLT1. (a, b) Selectivity plots of FRT1 (a) and MLT1 (b) measured by recording currents in the presence of different sugars in $5 \mathrm{mM}$ concentration (abbreviations as in Fig. 1). Results obtained with $-50 \mathrm{mV}$ test voltage are shown. Points present results obtained from individual oocytes and bars their mean $(n=3)$. $(\mathbf{c}, \mathbf{d})$ D-fructose transport kinetics of FRT1 (c) and D-glucose transport kinetics of MLT1 (d) at - $50 \mathrm{mV}$. Representative experiments are shown. Lines present Michaelis Menten kinetics prediction and the $K_{\mathrm{m}}$ values present mean and standard deviation from individual oocytes ( $n=6$ for Trire2_50618, $n=4$ for Trire2_67469). The figure was created with ggplot2 package for $\mathrm{R}^{79,85}$.

detailed selectivity studies indicated that these two transporters appeared to be specific for their single substrates (Fig. 5a,b). The currents elicited by FRT1 and MLT1 were lower than those elicited by the other transporters at the $5 \mathrm{mM}$ concentration used in the initial screening. This observation was explained by kinetics measurements, from which we discovered that these transporters have low affinities for their substrates (Fig. 5c,d). Regarding voltage-dependence of kinetic parameters, the $K_{\mathrm{m}}$ values as a function of voltage showed opposite trends for FRT1 and MLT1 (Supplementary Figure S5), and the maximum transport rate by FRT1 was more dependent on voltage than that of MLT1 (Supplementary Figure S6).

The discovery of these new sugar transporters provides further information about the sugar transport system of T. reesei. Interestingly, the affinities observed for these transporters are lower than what have typically been observed for fungal hexose transporters (Supplementary Table S2), although low/moderate-affinity yeast hexose transporters have $K_{\mathrm{m}}$ values in this range ${ }^{5}$. The observed low affinities might indicate that $\mathrm{D}$-fructose and $D$-glucose are not the main substrates of these transporters. Regarding MLT1, this hypothesis is supported by its homology to $\alpha$-linked disaccharide transporters (Fig. 1) and by its upregulation in the absence of CRE1 (Table 1). Interestingly, two putative maltose transporters (Trire2_65191 and_59388) have been either lost or mutated in T. reesei hyperproducer strain RUT-C30, which grows poorly on maltose ${ }^{50,51}$. The same strain has also a mutation in regulator BglR, which is involved in both $\mathrm{D}$-glucose and maltose utilization ${ }^{52}$. However, the improved growth of RUT-C30 on D-glucose is not consistent with BglR-negative phenotype $\mathrm{e}^{50,52}$, which could indicate that BglR is still functional in this strain, and that the poor growth on maltose is indeed caused by defects in maltose transport.

FRT1, on the other hand, had some homology to characterized D-fructose transporters (Fig. 1), which themselves are homologs of the Saccharomyces pastorianus $\mathrm{D}$-fructose/L-sorbose symporter Fsy ${ }^{53-55}$. However, as shown in Supplementary table S2, its affinity for D-fructose is lower than what has been observed for these transporters (Botrytis cinerea FRT1, Aspergillus niger 296054 and $1186134^{53,54}$ ). In Fig. 1, FRT1 is grouped under different clade than these transporters, as was also previously shown for An02g07610 ${ }^{56}$, which is in the same clade as FRT1 in Fig. 1. An02g07610 has been found to be upregulated in response to pectin-related carbon sources (D-galacturonic acid, L-rhamnose, D-galactose, L-arabinose), although one report indicated that it functions as a sucrose transporter in yeast ${ }^{56,57}$. The observed specificity of FRT1 for D-fructose, an easily metabolizable monosaccharide, is interesting since FRT1 has consistently been found to be upregulated in cellulase-inducing conditions $^{22,36,58}$ and to be downregulated in the presence of D-glucose in CRE1-dependent manner ${ }^{29,43}$. Additionally, FRT1 has also been found to be differentially phosphorylated in response to cellulase-induction ${ }^{59}$.

Uronic acid transporters Trire2_106330 (GAT1) and Trire2_69026 (GAT2) prefer different substrates. Our initial screening identified two transporters capable of transporting D-galacturonic and D-glucuronic acids. D-Galacturonic acid is the primary component of the plant cell wall polysaccharide pectin. Pectin-rich sidestreams from e.g. fruit processing industry have gained interest as feedstock for bioprocesses, since they are available in large quantities and contain lower amounts of lignin than lignocellulose biomass ${ }^{14}$. D-Glucuronic acid, on the other hand, is present in the hemicellulose fraction of lignocellulose biomass. Both of 
a
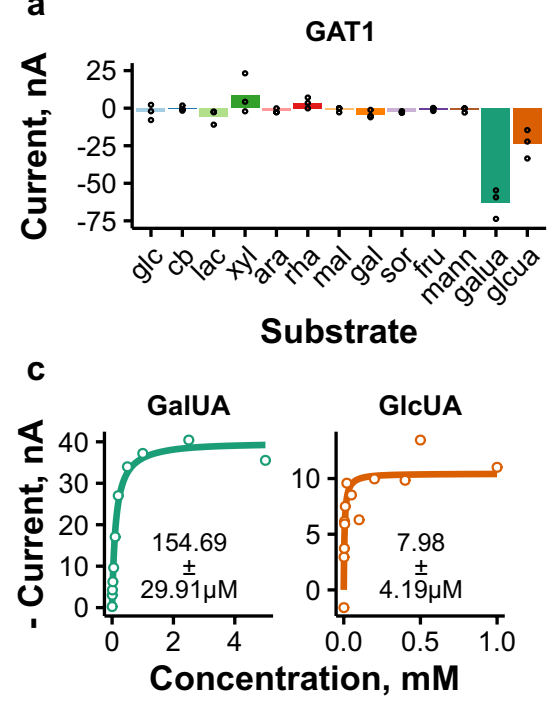

b
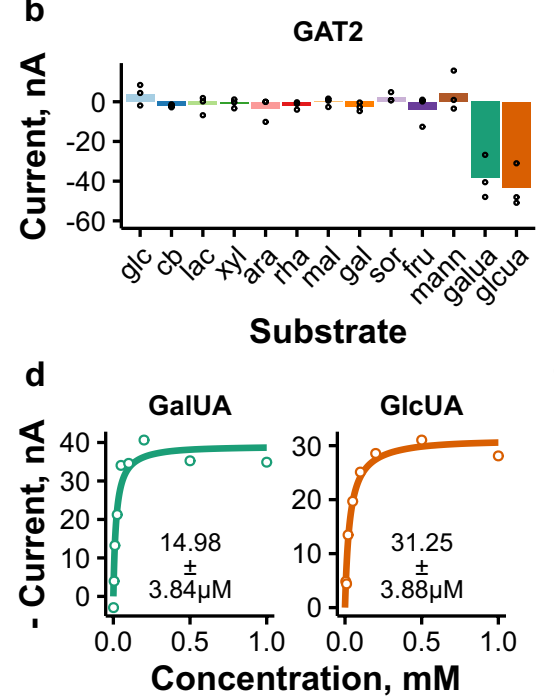

e
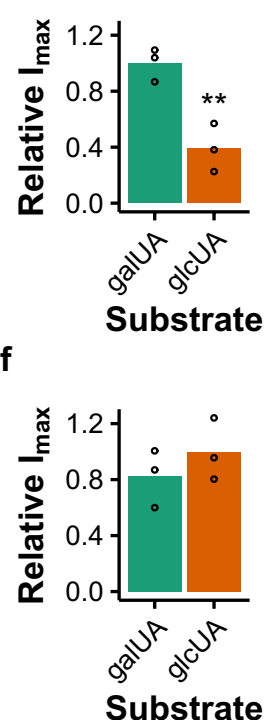

Figure 6. Electrophysiological characterization of uronic acid transporters GAT1 and GAT2. (a, b) Selectivity plots of GAT1 (a) and GAT2 (b) measured by recording currents in the presence of different sugars and sugar acids in $5 \mathrm{mM}$ concentration (abbreviations as in Fig. 1). Results obtained with $-50 \mathrm{mV}$ test voltage are shown. Points present results obtained from individual oocytes and bars their mean $(n=3)$. (c,d) Sugar acid transport kinetics of GAT1 (c) and GAT2 (d) at - $50 \mathrm{mV}$. Representative experiments are shown. Lines present the Michaelis Menten kinetics prediction and the $K_{\mathrm{m}}$ values present the mean and standard deviation from individual oocytes $(n=3)$. (e, f) Relative $I_{\max }$ values for GAT1 (e) and GAT2 (f) at $-50 \mathrm{mV}$. $I_{\max }$ values from individual oocytes were normalized to the mean $I_{\max }$ value of the substrate with the highest $I_{\max }$ value for each transporter. Points present results from individual oocytes and bars their mean $(n=3)$. Significance was estimated with Student's $t$-test $\left({ }^{*}: p<0.01\right)$. The figure was created with ggplot2 package for $\mathrm{R}^{79,85}$.

these transporters had been identified in a previous study, but apart from identifying their transport activity in yeast, no characterization had been done on them ${ }^{14}$. Since our initial screening identified them to be functional in oocytes, we set out to investigate them more thoroughly.

The two uronic acid transporters seemed to be specific for their substrates (Fig. 6a,b), with D-galacturonic acid inducing higher currents in Trire2_106330 (here named GAT1, for galacturonic acid transporter 1) than D-glucuronic acid. Kinetics experiments indicated that GAT1 has higher affinity for D-glucuronic acid than for D-galacturonic acid (Fig. 6c). Conversely Trire2_69026 (here named GAT2) has higher affinity for D-galacturonic acid, but the difference is not as large as with GAT1 (Fig. 6d). GAT1 had significantly higher $I_{\max }$ value for D-galacturonic than for D-glucuronic acid (Fig. 6e), in contrast to GAT2 for which the $I_{\max }$ values were not significantly different (Fig. $6 \mathrm{f}$ ). Of the transporters studied here, GAT1 was the only one which had significantly different $I_{\max }$ values for different substrates across all test voltages. This difference might allow the transport activity to be tuned to different sugar acids released from different plant cell wall polysaccharides. Regarding voltage-dependence of the kinetics, GAT2 was discovered to be more voltage-dependent than GAT1 (Supplementary Figure S6), and its affinity was found to decrease as the voltage became more depolarized (Supplementary Figure S5).

To our knowledge, only two fungal uronic acid transporters have been kinetically characterized in the literature, although kinetic parameters are only available for D-galacturonic acid ${ }^{14,60}$. The affinities of GAT1 and GAT2 for D-galacturonic acid lie between the previously characterized transporters from N. crassa and A. niger (Supplementary Table S3). For the first time, two uronic acid transporters have been characterized from the same fungal species and interestingly they had different preferences for D-galacturonic and D-glucuronic acids. More studies would be needed to investigate if they are regulated differently, since these sugar acids are found from different plant cell wall polysaccharides. Although pectinolytic regulators (such as GaaX, GaaR and RhaR of Aspergilli ${ }^{61}$ ) have not yet been characterized from T. reesei, $\mathrm{L}$-arabinose/D-galactose regulator ARA1 has been shown to regulate genes related to both hemicellulase and pectin metabolism, as $\mathrm{L}$-arabinose is present in both polymers $^{41}$.

\section{Conclusions}

The purpose of our study was to gain better understanding of the sugar transporter repertoire of T. reesei. Phylogenetic analysis was used to identify a reasonably sized set of transporters for the functional analysis, which provided a substantial amount of new information about T. reesei sugar transporters. All in all, transport activity was described for the first time for three transporters (GLT1, FRT1, MLT1), and we were also able to identify many new functionalities for transporters which had been identified previously. 
The studied set of transporters was diverse, and we could identify transporters for all the different classes of sugars mentioned in Fig. 1, except for $\alpha$-linked disaccharides. It is possible that some of the transporters for which we didn't detect transport activity might still exhibit it. There could have been problems with protein expression, folding or insertion to the membrane, but we did not address or try to troubleshoot these possibilities. Some membrane transporters require ancillary proteins to function or to be correctly localized ${ }^{62,63}$, but to our knowledge this has not been observed in fungal sugar transporters. There are also many possible substrates that we didn't analyze (e.g. polyols). Additionally, some transporters appear to exhibit their activity only on a certain concentration range, which we might have missed $^{64}$.

Besides providing information about the physiology of the fungus, these results could have practical applications. For example, deletion of D-glucose transporter(s) has resulted in increased protein production in both $N$. crassa and T. reese $i^{8,19}$. Double deletion of high-affinity D-glucose transporters STP1 and STR3 could be attempted for this purpose, as has been done in $N$. crassa ${ }^{8}$. Since T. reesei has been modified to produce high amounts of protein with $\mathrm{D}$-glucose as the carbon source ${ }^{65-67}$, overexpression of GLT1 could be also attempted to increase the protein production in these strains. Nevertheless, protein production is conventionally done on lactosebased media, and thus manipulation of $\beta$-linked disaccharide transport could be also attempted. As a practical example, overexpression of CRT1 has been indeed shown to result in earlier onset of cellulase production or in higher cellulase expression ${ }^{19,21,23}$.

Regarding transport kinetics, the majority of the identified transporters exhibited high or moderate affinity for their primary substrates. Exceptions were MLT1 and FRT1, which had considerably low affinity when compared to other fungal transporters capable of transporting these sugars (Supplementary Table S2). As in N. crassa $a^{8}$, the D-glucose transport system of T. reesei appears to include high-affinity (STP1, STR3) and low/moderate-affinity (GLT1) transporters. In addition to these transporters, three additional D-glucose transporters have been identified from T. reesei (STR $1^{7,17}$, STR $2^{7}$, XLTR $1^{18}$ ), but their expression profile does not support their involvement in the bulk of $\mathrm{D}$-glucose transport.

This study, along with two previously published studies ${ }^{33,34}$, employed X. laevis oocytes for the electrophysiological characterization of fungal sugar transporters. Although there were differences in the $K_{\mathrm{m}}$ values determined in yeast and oocytes, the general trend (low or high affinity) was similar between both as shown in Supplementary Figure S9. These differences could be caused by differences in membrane potential or in the lipid composition of the cell membrane. Since many of the characterized fungal sugar transporters utilize the proton symport mechanism (Supplementary Tables S2 and S3), they could be studied further with this system. Additionally, since the genomes of saprophytic fungi contain many uncharacterized sugar transporters, electrophysiological methods could be used for the identification of transport activities from the thus far unknown transporters. Since robotic systems for oocyte injection and TEVC analysis are available ${ }^{68}$, high-throughput screening would be also possible.

\section{Materials and methods}

Microbial media, cultivation and chemicals. Yeast strains were cultured in YP (1\% yeast extract, $2 \%$ peptone) or synthetic complete ( $0.67 \%$ yeast nitrogen base without amino acids, SC-Ura drop-out mix) medium with $2 \%$ glycerol and $2 \%$ ethanol as carbon sources unless otherwise mentioned ${ }^{69}$. Both media were supplemented with $2 \%$ agar for preparing solid media. Yeast was grown in $30^{\circ} \mathrm{C}$ with $230 \mathrm{rpm}$ shaking in $20 \mathrm{~mL}$ volume in $100 \mathrm{~mL}$ flasks or in $50 \mathrm{~mL}$ volume in $250 \mathrm{~mL}$ flasks. Growth curves were recorded with Bioscreen C incubator (Oy Growth Curves Ab, Helsinki, Finland) as described before ${ }^{21}$.

Chemicals were obtained from Merck KGaA (Darmstadt, Germany) and molecular biology reagents from Thermo Fisher Scientific (Waltham, MA, USA) unless otherwise mentioned. Lactose was obtained from VWR (Helsinki, Finland), and L-rhamnose and L-sorbose were obtained from Fluka (Charlotte, NC, USA). Cellotriose and cellotetraose were obtained from Carbosynth (Compton, Berkshire, UK) and $\alpha$-sophorose from SERVA Electrophoresis GmbH (Heidelberg, Germany).

Molecular biology methods. High-fidelity PCR was done with KAPA HiFi polymerase (Roche, Basel, Switzerland) and colony PCR with DreamTaq polymerase. Restriction digestions were performed with restriction enzymes from Thermo Fisher Scientific or NEB (Ipswich, MA, USA). E. coli transformations were done with electroporation and yeast transformations with the LiAc/ssDNA/PEG method described by Gietz and Woods ${ }^{70}$. In vitro transcription was done with mMessage mMachine T7 kit. Primers were obtained from Thermo Fisher Scientific and they are listed in Supplementary Table S4. Escherichia coli strain Top10 was used for cloning and for storing the plasmids.

Expression of transporters in yeast. For the yeast expression, synthetic genes were obtained with codon-optimization for yeast (GeneArt, Thermo-Scientific), with the exception of Trire2_69026 which was obtained from T. reesei cDNA. The amino acid sequences for gene synthesis were obtained from the T. reesei QM6a genome annotation (version 2.0) ${ }^{71}$, except for Trire2_62380 (STR3), _3405 (CRT1),_67752 and_67469. For Trire2_62380,_3405 and _67752, we used the genes from the RUT-C30 genome assembly ${ }^{72}$ (TrireRUTC30_95062,_109243, and_79984, respectively). RUT-C30 versions of Trire2_3405 and_67752 have been shown to be functional in previous studies ${ }^{20,21}$. Sequence for Trire2_67469 was manually annotated based on a homolog identified from other species of Trichoderma, as shown in Supplementary Figure S10a. Similarly, we identified an alternative version for Trire2_56684, which was expressed in addition to the QM6a version (Supplementary Figure S10b). For Trire2_72383, we expressed both RUT-C30 and QM6a version. All constructs are listed in Supplementary Table S5.

The synthetic genes were cloned to yeast expression vector (URA3, CEN6/ARS4, PGK1 promoter, ENO1 terminator) with the yeast MoClo toolkit (parts MoClo parts pYTK-2, -11, -51, -67, -74, -81, -84) ${ }^{73}$. The resulting 
constructs were sequenced with primers SaSS-19 and -20. The gene coding for Trire2_69026 was amplified from T. reesei QM6a cDNA with primers SaSS-75-76, and ligated to B2159 vector (derived from pYX212 as described in Ref. ${ }^{74}$ ) which had been digested with EcoRI and BamHI. The resulting construct was sequenced with primers TPI1_P_long and TPI1_T_long.

The expression plasmids were transformed into yeast strain ySS1, which as an EBY.VW5000 derivative lacks the endogenous hexose transporters and additionally expresses $N$. crassa intracellular $\beta$-glucosidase GH1-1 ${ }^{21}$. ySS1 was pre-grown on YPGE and the transformation reactions were plated on SCGE-Ura medium.

Construction of plasmids for $\boldsymbol{X}$. laevis expression. To express the synthetic genes in X. laevis oocytes, the oocyte expression vector Pol $1^{75}$ was modified to be compatible with the yeast MoClo system (Havukainen et al., submitted). The MoClo-compatible version of Poll was digested with BsaI and the two resulting fragments (Poll contains one endogenous BsaI site) were gel purified. The backbone fragments were ligated with T4 ligase (NEB) with the genes which were liberated from the original GeneArt pMA-T plasmids with BsaI. The constructs were sequenced with primers SaSS-57 primer and T7 promoter primer.

The genes coding for Trire2_69026,_72383,_77517 and _79202 were obtained from T. reesei cDNA for X. laevis expression. Trire2_69026 was amplified from plasmid pSS87 (Supplementary Table S5) with primers SaSS95-96 and the amplicon was digested with BamHI and EcoRI and ligated to Poll vector digested with the same enzymes. The genes coding for Trire2_72383,_77517 and_79202 were amplified from cDNA prepared from T. reesei QM6a with primers PP-203-204 and PP-209-212. Trire2_72383 amplicon was digested with BamHI and HindIII and ligated to $X$. laevis expression vector pMJB08 (pol1 derivative with various tags ${ }^{76}$ ) which had been digested with the same enzymes. Trire2_77517 and_79202 expression vectors were constructed similarly, except that for Trire2_77517 the amplicon and vector were cut with BamHI and EcoRI, and for Trire2_79202 they were cut with XbaI and HindIII. We noted that the Trire2_79202 sequence obtained from cDNA differed from the QM6a sequence in that the second intron was missing (Supplementary Figure S10c).

Preparation of mRNA and $X$. laevis oocyte injection. Oocytes were obtained via surgery as described by Clemencon et al. ${ }^{77}$. Briefly, adult female $X$. laevis frogs were anaesthetized by immersion in ice/water slurry containing $1 \mathrm{~g} / \mathrm{L} 3$-aminobenzoate methanesulfonate and the oocytes were removed with surgery. After removing the oocytes, the cuts were sutured, and after recovering from anesthesia the frog was placed in isolation tank to recover for 1 week. During the studies, each of the used frogs was subjected to surgery only once. All experiments using the X. laevis animals were in accordance with the Swiss Animal Welfare law, reported according to applicable ARRIVE guidelines ${ }^{78}$ and approved by the local Veterinary Authority (Amt für Veterinärwesen Kantons Bern; Permit Number: BE60/2018).

After the surgery, the oocytes were suspended in modified Barth's medium with $\mathrm{Ca}^{2+}\left(\mathrm{MBM}+\mathrm{Ca}^{2+} ; 88 \mathrm{mM}\right.$ $\mathrm{NaCl}, 1 \mathrm{mM} \mathrm{KCl}, 2.4 \mathrm{mM} \mathrm{NaHCO}_{3}, 1.57 \mathrm{mM} \mathrm{MgSO}_{4} .7 \mathrm{H}_{2} \mathrm{O}, 0.66 \mathrm{mM} \mathrm{NaNO}_{3}, 0.75 \mathrm{mM} \mathrm{CaCl}_{2}, 10 \mathrm{mM} \mathrm{HEPES}$ which was supplemented with penicillin and streptomycin. The oocyte sacs were separated to 3-4 mm pieces with forceps. Oocytes were defolliculated by washing with $\mathrm{MBM}-\mathrm{Ca}^{2+}\left(\mathrm{MBM}+\mathrm{Ca}^{2+}\right.$ without $\left.\mathrm{CaCl}_{2}\right)$, after which they were suspended to MBM-Ca ${ }^{2+}$ with $4 \mathrm{~g} / \mathrm{L}$ collagenase (NB4, SERVA Electrophoresis GmbH, Heidelberg, Germany) and incubated in rocking shaker for 1 hour. After the incubation, the washing and collagenase incubation steps were repeated. Finally the oocytes were washed with $\mathrm{MBM}+\mathrm{Ca}^{2+}$ and healthy stage V-VI oocytes were picked.

The pol1-based plasmids containing the transporter CDSs were linearized with NheI and in vitro transcribed into mRNA. After the transcription the product was purified with $\mathrm{LiCl}$ precipitation according to the kit instructions. The oocytes were injected with 10-30 ng mRNA in $50 \mathrm{~nL}$ volume or with the same volume of water. The injection was done with Nanoject II microinjector (Drummond Scientific, Broomall, PA, USA). After injection the oocytes were suspended in $\mathrm{MBM}+\mathrm{Ca}^{2+}$ and incubated in $17^{\circ} \mathrm{C}$ for 3-7 days.

Electrophysiology experiments with $\boldsymbol{X}$. laevis oocytes. Two-electrode voltage clamp (TEVC) technique was used for the electrophysiological characterization. In this method, the membrane potential of the oocyte is clamped to a specific voltage and the transport is measured by recording the substrate-induced changes in the membrane current ${ }^{32}$. The measurements were done on a TEVC setup consisting of 2-channel perfusion system, OC-725C Oocyte clamp amplifier (Warner Instruments, Hamden, CT, USA) and Axon Digidata 1440A digitizer (Molecular Devices, San Jose, CA, USA). The system was calibrated with oocyte model cell. Microelectrodes filled with $3 \mathrm{M} \mathrm{KCl}$ and with resistance between $0.5-5 \mathrm{M} \Omega$ were used. pClamp software suite (version 10.2, Molecular Devices, https://www.moleculardevices.com/) was used for the analysis. ND-96 buffer without sodium (100 mM choline, $2 \mathrm{mM} \mathrm{KCl,} 1 \mathrm{mM} \mathrm{CaCl}_{2} \cdot 2 \mathrm{H}_{2} \mathrm{O}, 1 \mathrm{mM} \mathrm{MgCl} \mathrm{m}_{2} \cdot 6 \mathrm{H}_{2} \mathrm{O}, 3 \mathrm{mM}$ HEPES) was used for the experiments. Each experiment was done with at least three oocytes derived from at least two different frogs, except for some sugars in CRT1 and CDT-1 selectivity experiments where only 2 oocytes were used. Each transporter was tested at least with 11 different sugars, including monosaccharides and disaccharides (glc, fru, gal, mann, rha, sor, xyl, ara, cb, lac, mal; abbreviations as in Fig. 1).

For the current trace recordings, the oocytes were perfused with ND-96 (pH 7.4) and clamped at $-50 \mathrm{mV}$. For each sugar, the oocyte was first perfused with ND-96 (pH 7.4) until the current stabilized, then with ND-96 ( $\mathrm{pH}$ 5.5) until the current stabilized and finally with ND-96 (pH 5.5) containing $5 \mathrm{mM}$ sugar. Afterwards the current was recovered to baseline by perfusing with ND-96 ( $\mathrm{pH} 7.4$ ). The resulting current traces were recorded with Axoscope program of the pClamp software suite.

The I-V curves were recorded with Clampex program of the pClamp software suite. The oocytes were clamped at $-50 \mathrm{mV}$, perfused with the test solution until the current stabilized and then $200 \mathrm{~ms}$ step-wise changes of membrane potential from $-150 \mathrm{mV}$ to $50 \mathrm{mV}$ were applied. For each measurement, a recording was done first 
with buffer alone and then with the same buffer which contained the sugar to be analyzed. The steady-state currents obtained in the absence of sugar were subtracted from the currents obtained in the presence of sugar for each of the tested voltages. Between measurements the oocyte was stabilized by perfusing with $\mathrm{pH} 7.4$ buffer.

The selectivity was measured by recording I-V curves with $5 \mathrm{mM}$ substrate solutions prepared in ND-96 pH 5.5. Sugar acid solutions were adjusted to $\mathrm{pH} 5.5$ with $\mathrm{KOH}$. For the kinetics experiments I-V curve recordings were done with different sugar concentrations with $\mathrm{pH} 5.5$ buffer. For the $\mathrm{pH}$-dependence experiments of CDT-1 and CRT1, I-V curve recordings were done with ND-96 (with $5 \mathrm{mM}$ cellobiose) adjusted to different $\mathrm{pH}$ values. The kinetics parameters for sugars and protons were calculated by fitting the currents as a function of substrate concentration to Michaelis-Menten equation (Eq. 1), where $I_{\max }=$ maximum current, $[S]=$ substrate concentration and $K_{m}=$ Michaelis constant. The fitting was done with the n Is function with the default Gauss-Newton algorithm. The nls function belongs to package stats which is part of $\mathrm{R}^{79}$. The $K_{\mathrm{m}}$ values are presented as mean \pm standard deviation from at least three different oocytes. For the analysis of voltage-dependence of kinetics shown in Supplementary Figures S4, S6 and S8, we normalized the currents to the highest negative current obtained at $-50 \mathrm{mV}$ during the experiment.

$$
I=\frac{I_{\max }[S]}{[S]+K_{m}}
$$

Uptake experiments. Uptake experiments were done as previously ${ }^{21}$. Briefly, ySS1-based yeast strains were inoculated into $20 \mathrm{~mL}$ SCGE-Ura medium and grown for 2 days. Then the strains were inoculated into $50 \mathrm{~mL}$ of the same medium and grown into exponential phase. Cells were harvested by centrifugation, washed

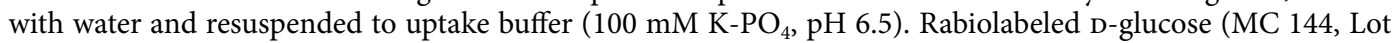
945-006-256-A-20180329-DNG, Moravek Biochemicals, Brea, CA, USA) was diluted with ordinary sugar to reach the desired concentration and radiospecific activity. Aliquots of the yeast suspensions and the label were incubated 5-10 $\mathrm{min}$ at $28^{\circ} \mathrm{C}$ before starting the reaction. Reaction mixture consisted of $40 \mu \mathrm{L}$ cell suspension and $20 \mu \mathrm{L}$ of the label in a cone-shaped glass tube. Reaction was stopped after desired time by adding $10 \mathrm{~mL}$ icecold water to the reaction and filtering the resulting mixture. The tube was washed with same amount of water, which was also filtered. The filter containing the yeast was suspended to $4 \mathrm{~mL}$ of Ultima Gold XR liquid scintillation cocktail (PerkinElmer, Waltham, MA, USA). The samples were counted with TriCarb 2810 TR scintillation counter (PerkinElmer). Blank samples were measured similarly, except that $10 \mathrm{~mL}$ water was added to yeast suspension before the label, and after the addition of the label the mixture was filtered immediately. The chosen reaction times were ensured to be on the part of the curve which was linear in respect to time.

Uptake rate was determined by substracting the counts (as counts per minute, cpm) obtained for the blank samples from the actual samples, and by dividing the blank-corrected counts with the radiospecific activity of the label $(\mathrm{cpm} / \mathrm{nmol})$, the amount of yeast used (OD) and by the reaction time ( $\mathrm{min})$. OD values were further converted to CDW values using a previously published correlation factor for ySS1 ${ }^{21}$. D-glucose uptake kinetics were fitted to equation 2 individually for each biological replicate $(n=3)$, and the values are presented as mean \pm standard deviation from these fits. The negative control strain expressed $T$. reese $i$ intracellular $\beta$-glucosidase CEL1a from a similar expression plasmid ${ }^{21}$.

$$
V=\frac{V_{\max }[S]}{[S]+K_{m}}
$$

Phylogenetic analysis. Published fungal sugar transporters and putative T. reesei sugar transporters were selected for the phylogenetic analysis. A List of putative T. reesei sugar transporters was obtained from Ref. ${ }^{1}$. From this list we excluded some transporters based on their transporter classification database (TCDB) family ${ }^{80}$, if the description indicated that the predicted substrate was not sugar, polyol or quinate (Trire2_106118,_121441, _44175,_45852,_77552,_81389,_45868,_60988,_82037). Some were further exluded based on transmembrane prediction, which indicated that they didn't possess the characteristic 12 transmembrane domain topology of major facilitator superfamily transporters (Trire2_5890,_75021,_80058,_61278,_60086). Additionally, we included putative transporter Trire2_79202 since it has been hypothesized to be a lactose transporter ${ }^{28}$.

The amino acid sequences of the transporters were obtained from GenBank or Uniprot. Their accession numbers with references for the transported substrates are listed in in Supplementary Table S1. We did not consider deletion phenotypes alone as definite evidence of transport function (as with e.g. Tr_HXT1 ${ }^{6}$, Nc_SUT-28 ${ }^{27}$ ), and thus these substrates were not included in the figure. Sequence alignment was done with MUSCLE ${ }^{81}$, and the phylogenetic analysis was performed with maximum likelihood method with 100 bootstrap runs with partial gap removal (95\% site coverage cutoff $)^{82}$. Both steps were performed with MEGA X software (version 10.1.1) ${ }^{83}$, and the tree was visualized with ape package for $\mathrm{R}^{84}$.

\section{Data availability}

The datasets generated during the current study are available from the corresponding author on reasonable request.

Received: 23 March 2021; Accepted: 21 June 2021

Published online: 19 July 2021 


\section{References}

1. Nogueira, K. M. V. et al. Sugar transporters from industrial fungi: Key to improving second-generation ethanol production. Renew. Sustain. Energy Rev. 131, 109991 (2020).

2. Pao, S. S., Paulsen, I. T. \& Saier, M. H. Major facilitator superfamily. Microbiol. Mol. Biol. Rev. 62, 1-34 (1998).

3. Podolsky, I. A., Seppälä, S., Xu, H., Jin, Y.-S. \& O’Malley, M. A. A SWEET surprise: anaerobic fungal sugar transporters and chimeras enhance sugar uptake in yeast. Metab. Eng. 66, 137-147 (2021).

4. Chen, L.-Q., Cheung, L. S., Feng, L., Tanner, W. \& Frommer, W. B. Transport of sugars. Annu. Rev. Biochem. 84, 865-894 (2015).

5. Bisson, L. F., Fan, Q. \& Walker, G. A. Sugar and glycerol transport in Saccharomyces cerevisiae. In Ramos, J., Sychrova, H. \& Kschischo, M. (eds.) Yeast Membrane Transport, Advances in Experimental Medicine and Biology, vol. 892, 125-168 (Springer, Cham, Switzerland, 2016)

6. Ramos, A. S. et al. Oxygen-and glucose-dependent expression of Trhxt1, a putative glucose transporter gene of Trichoderma reesei. Biochemistry 45, 8184-8192 (2006).

7. Sloothaak, J. et al. Identification and functional characterization of novel xylose transporters from the cell factories Aspergillus niger and Trichoderma reesei. Biotechnol. Biofuels 9, 148 (2016).

8. Wang, B. et al. Identification and characterization of the glucose dual-affinity transport system in Neurospora crassa: pleiotropic roles in nutrient transport, signaling, and carbon catabolite repression. Biotechnol. Biofuels 10, 17 (2017).

9. Sloothaak, J. et al. Aspergillus niger membrane-associated proteome analysis for the identification of glucose transporters. Biotechnol. Biofuels 8, 150 (2015).

10. Vankuyk, P. A. et al. Aspergillus niger mstA encodes a high-affinity sugar $/ \mathrm{H}^{+}$symporter which is regulated in response to extracellular pH. Biochem. J. 379, 375-383 (2004).

11. Lingner, U., Münch, S., Deising, H. B. \& Sauer, N. Hexose transporters of a hemibiotrophic plant pathogen functional variations and regulatory differences at different stages of infection. J. Biol. Chem. 286, 20913-20922 (2011).

12. Maier, A., Völker, B., Boles, E. \& Fuhrmann, G. F. Characterisation of glucose transport in Saccharomyces cerevisiae with plasma membrane vesicles (countertransport) and intact cells (initial uptake) with single Hxt1, Hxt2, Hxt3, Hxt4, Hxt6, Hxt7 or Gal2 transporters. FEMS Yeast Res. 2, 539-550 (2002).

13. Nijland, J. G. \& Driessen, A. J. Engineering of pentose transport in Saccharomyces cerevisiae for biotechnological applications. Front. Bioeng. Biotechnol. 7, 464 (2020).

14. Protzko, R. J. et al. Engineering Saccharomyces cerevisiae for co-utilization of D-galacturonic acid and D-glucose from citrus peel waste. Nat. Commun. 9, 1-10 (2018).

15. Hahn-Hägerdal, B., Karhumaa, K., Fonseca, C., Spencer-Martins, I. \& Gorwa-Grauslund, M. F. Towards industrial pentosefermenting yeast strains. Appl. Microbiol. Biotechnol. 74, 937-953 (2007).

16. Chaudhary, N., Kumari, I., Sandhu, P., Ahmed, M. \& Akhter, Y. Proteome scale census of major facilitator superfamily transporters in Trichoderma reesei using protein sequence and structure based classification enhanced ranking. Gene 585, 166-176 (2016).

17. Huang, Z.-B. et al. A novel major facilitator transporter TrSTR1 is essential for pentose utilization and involved in xylanase induction in Trichoderma reesei. Biochem. Biophys. Res. Commun. 460, 663-669 (2015).

18. Jiang, Y. et al. Identification and characterization of an efficient D-xylose transporter in Saccharomyces cerevisiae. J. Agric. Food Chem. 68, 2702-2710 (2020).

19. Zhang, W. et al. Two major facilitator superfamily sugar transporters from Trichoderma reesei and their roles in induction of cellulase biosynthesis. J. Biol. Chem. 288, 32861-32872 (2013).

20. Casa-Villegas, M., Polaina, J. \& Marín-Navarro, J. Cellobiose fermentation by Saccharomyces cerevisiae: Comparative analysis of intra versus extracellular sugar hydrolysis. Process Biochem. 75, 59-67 (2018).

21. Havukainen, S., Valkonen, M., Koivuranta, K. \& Landowski, C. P. Studies on sugar transporter CRT1 reveal new characteristics that are critical for cellulase induction in Trichoderma reesei. Biotechnol. Biofuels 13, 1-20 (2020).

22. Ries, L. et al. Genome-wide transcriptional response of Trichoderma reesei to lignocellulose using RNA sequencing and comparison with Aspergillus niger. BMC Genomics 14, 541 (2013).

23. Ivanova, C., Bååth, J. A., Seiboth, B. \& Kubicek, C. P. Systems analysis of lactose metabolism in Trichoderma reesei identifies a lactose permease that is essential for cellulase induction. PLoS one 8, e62631 (2013).

24. dos Santos Castro, L. et al. Understanding the role of the master regulator XYR1 in Trichoderma reesei by global transcriptional analysis. Front. Microbiol. 7, 175 (2016).

25. Zhang, J. et al. The transcription factor ACE3 controls cellulase activities and lactose metabolism via two additional regulators in the fungus Trichoderma reesei. J. Biol. Chem. 294, 18435-18450 (2019).

26. Amore, A., Giacobbe, S. \& Faraco, V. Regulation of cellulase and hemicellulase gene expression in fungi. Curr. Genomics 14, 230-249 (2013).

27. Wu, V. W. et al. The regulatory and transcriptional landscape associated with carbon utilization in a filamentous fungus. Proc. Natl. Acad. Sci. USA 117, 6003-6013 (2020).

28. Porciuncula, JDO et al. Identification of major facilitator transporters involved in cellulase production during lactose culture of Trichoderma reesei PC-3-7. Biosci. Biotechnol. Biochem. 77, 1014-1022 (2013).

29. Antoniêto, A. C. C., dos Santos Castro, L., Silva-Rocha, R., Persinoti, G. F. \& Silva, R. N. Defining the genome-wide role of CRE1 during carbon catabolite repression in Trichoderma reesei using RNA-Seq analysis. Fungal Genet. Biol. 73, 93-103 (2014).

30. Paloheimo, M., Haarmann, T., Mäkinen, S. \& Vehmaanperä, J. Production of industrial enzymes in Trichoderma reesei. In Schmoll, M. \& Christoph, D. (eds.) Gene Expression Systems in Fungi: Advancements and Applications, Fungal Biology, 23-57 (Springer, Cham, Switzerland, 2016).

31. Miller, A. \& Zhou, J. Xenopus oocytes as an expression system for plant transporters. Biochim. Biophys. Acta Biomembr. 1465, 343-358 (2000).

32. Guan, B., Chen, X. \& Zhang, H. Two-electrode voltage clamp. In Gamper, N. (ed.) Ion Channels: Methods and Protocols, Methods in Molecular Biology, vol. 998, 79-89 (Springer, New York, NY, USA, 2013).

33. Wittek, A. et al. The fungal UmSrt1 and maize ZmSUT1 sucrose transporters battle for plant sugar resources. J. Integr. Plant Biol. 59, 422-435 (2017).

34. Voegele, R. T., Struck, C., Hahn, M. \& Mendgen, K. The role of haustoria in sugar supply during infection of broad bean by the rust fungus Uromyces fabae. Proc. Natl. Acad. Sci. USA 98, 8133-8138 (2001).

35. Wright, E. M., Loo, D. D. \& Hirayama, B. A. Biology of human sodium glucose transporters. Physiol. Rev. 91, 733-794 (2011).

36. Bischof, R. et al. Comparative analysis of the Trichoderma reesei transcriptome during growth on the cellulase inducing substrates wheat straw and lactose. Biotechnol. Biofuels 6, 127 (2013).

37. Häkkinen, M. et al. Screening of candidate regulators for cellulase and hemicellulase production in Trichoderma reesei and identification of a factor essential for cellulase production. Biotechnol. Biofuels 7, 14 (2014).

38. Nogueira, K. M. et al. Characterization of a novel sugar transporter involved in sugarcane bagasse degradation in Trichoderma reesei. Biotechnol. Biofuels 11, 84 (2018).

39. Kim, H., Lee, W.-H., Galazka, J. M., Cate, J. H. \& Jin, Y.-S. Analysis of cellodextrin transporters from Neurospora crassa in Saccharomyces cerevisiae for cellobiose fermentation. Appl. Microbiol. Biotechnol. 98, 1087-1094 (2014). 
40. Herold, S., Bischof, R., Metz, B., Seiboth, B. \& Kubicek, C. P. Xylanase gene transcription in Trichoderma reesei is triggered by different inducers representing different hemicellulosic pentose polymers. Eukaryot. Cell 12, 390-398 (2013).

41. Benocci, T. et al. ARA1 regulates not only L-arabinose but also D-galactose catabolism in Trichoderma reesei. FEBS Lett. 592, 60-70 (2018).

42. Nogawa, M., Goto, M., Okada, H. \& Morikawa, Y. 1-Sorbose induces cellulase gene transcription in the cellulolytic fungus Trichoderma reesei. Curr. Genet. 38, 329-334 (2001).

43. Stappler, E., Dattenböck, C., Tisch, D. \& Schmoll, M. Analysis of light-and carbon-specific transcriptomes implicates a class of G-protein-coupled receptors in cellulose sensing. Msphere 2, e00089-17 (2017).

44. Wieczorke, R. et al. Concurrent knock-out of at least 20 transporter genes is required to block uptake of hexoses in Saccharomyces cerevisiae. FEBS Lett. 464, 123-128 (1999).

45. Wang, C. et al. Cloning and characterization of heterologous transporters in Saccharomyces cerevisiae and identification of important amino acids for xylose utilization. Metab. Eng. 30, 79-88 (2015).

46. Zhang, W. et al. Identification of residues important for substrate uptake in a glucose transporter from the filamentous fungus Trichoderma reesei. Sci. Rep. 5, 1-10 (2015).

47. Znameroski, E. A. et al. Evidence for transceptor function of cellodextrin transporters in Neurospora crassa. J. Biol. Chem. 289, 2610-2619 (2014).

48. Galazka, J. M. et al. Cellodextrin transport in yeast for improved biofuel production. Science 330, 84-86 (2010).

49. de Ruijter, J. C., Igarashi, K. \& Penttilä, M. The Lipomyces starkeyi gene Ls120451 encodes a cellobiose transporter that enables cellobiose fermentation in Saccharomyces cerevisiae. FEMS Yeast Res. 20, foaa019 (2020).

50. Seidl, V. et al. The Hypocrea jecorina (Trichoderma reesei) hypercellulolytic mutant RUT C30 lacks a $85 \mathrm{~kb}$ (29 gene-encoding) region of the wild-type genome. BMC Genomics 9, 1-15 (2008).

51. Le Crom, S. et al. Tracking the roots of cellulase hyperproduction by the fungus Trichoderma reesei using massively parallel DNA sequencing. Proc. Natl. Acad. Sci. USA 106, 16151-16156 (2009).

52. Nitta, M. et al. A new $\mathrm{Zn}(\mathrm{II})_{2} \mathrm{Cys}_{6}$-type transcription factor BglR regulates $\beta$-glucosidase expression in Trichoderma reesei. Fungal Genet. Biol.49, 388-397 (2012).

53. Coelho, M. A., Gonçalves, C., Sampaio, J. P. \& Gonçalves, P. Extensive intra-kingdom horizontal gene transfer converging on a fungal fructose transporter gene. PLoS Genet 9, e1003587 (2013).

54. Doehlemann, G., Molitor, F. \& Hahn, M. Molecular and functional characterization of a fructose specific transporter from the gray mold fungus Botrytis cinerea. Fungal Genet. Biol. 42, 601-610 (2005).

55. Gonçalves, P., de Sousa, H. R. \& Spencer-Martins, I. FSY1, a novel gene encoding a specific fructose $/ \mathrm{H}^{+}$symporter in the type strain of Saccharomyces carlsbergensis. J. Bacteriol. 182, 5628-5630 (2000).

56. Peng, M., Aguilar-Pontes, M. V., de Vries, R. P. \& Mäkelä, M. R. In silico analysis of putative sugar transporter genes in Aspergillus niger using phylogeny and comparative transcriptomics. Front. Microbiol. 9, 1045 (2018).

57. de Vries, R. P. et al. Comparative genomics reveals high biological diversity and specific adaptations in the industrially and medically important fungal genus Aspergillus. Genome Biol. 18, 1-45 (2017).

58. dos Santos Castro, L. et al. Comparative metabolism of cellulose, sophorose and glucose in Trichoderma reesei using high-throughput genomic and proteomic analyses. Biotechnol. Biofuels 7, 1-18 (2014).

59. Nguyen, E. V. et al. Quantitative site-specific phosphoproteomics of Trichoderma reesei signaling pathways upon induction of hydrolytic enzyme production. J. Proteome Res. 15, 457-467 (2016).

60. Benz, J. P. et al. Identification and characterization of a galacturonic acid transporter from Neurospora crassa and its application for Saccharomyces cerevisiae fermentation processes. Biotechnol. Biofuels 7, 20 (2014).

61. Benocci, T., Aguilar-Pontes, M. V., Zhou, M., Seiboth, B. \& Vries, R. P. Regulators of plant biomass degradation in Ascomycetous fungi. Biotechnol. Biofuels 10, 152 (2017).

62. Fairweather, S. J., Shah, N. \& Bröer, S. Heteromeric solute carriers: function, structure, pathology and pharmacology. In Atassi, M. Z. (ed.) Protein reviews, of Advances in Experimental Medicine and Biology, vol. 1314, 13-127 (Springer, Cham, Switzerland, 2020).

63. Kerr, I. D. \& Bennett, M. J. New insight into the biochemical mechanisms regulating auxin transport in plants. Biochem. J. 401, 613-622 (2007).

64. Paulsen, P. A., Custódio, T. F. \& Pedersen, B. P. Crystal structure of the plant symporter STP10 illuminates sugar uptake mechanism in monosaccharide transporter superfamily. Nat. commun. 10, 1-8 (2019).

65. Derntl, C. et al. Mutation of the Xylanase regulator 1 causes a glucose blind hydrolase expressing phenotype in industrially used Trichoderma strains. Biotechnol. Biofuels 6, 1-11 (2013).

66. Rantasalo, A. et al. A universal gene expression system for fungi. Nucleic Acids Res. 46, e111 (2018).

67. Luo, Y. et al. Modification of transcriptional factor ACE3 enhances protein production in Trichoderma reesei in the absence of cellulase gene inducer. Biotechnol. Biofuels 13, 1-16 (2020).

68. Schnizler, K., Küster, M., Methfessel, C. \& Fejtl, M. The roboocyte: automated cDNA/mRNA injection and subsequent TEVC recording on Xenopus oocytes in 96-well microtiter plates. Recept. Channels 9, 41-48 (2003).

69. Sherman, F., Getting started with yeast. In Guide to Yeast Genetics and Molecular and Cell Biology - Part B, Methods in Enzymology, vol. 350 (eds Guthrie, C. \& Fink, G. R.) 3-41 (Elsevier, 2002).

70. Gietz, R. D. \& Woods, R. A. Yeast transformation by the LiAc/SS Carrier DNA/PEG method. In Xiao, W. (ed.) Yeast Protocol, Methods in Molecular Biology, 107-120 (Springer, Saskatoon, Saskatchewan, Canada, 2006).

71. Martinez, D. et al. Genome sequencing and analysis of the biomass-degrading fungus Trichoderma reesei (syn Hypocrea jecorina). Nat. Biotechnol. 26, 553 (2008).

72. Jourdier, E. et al. Proximity ligation scaffolding and comparison of two Trichoderma reesei strains genomes. Biotechnol. Biofuels 10, 151 (2017).

73. Lee, M. E., DeLoache, W. C., Cervantes, B. \& Dueber, J. E. A highly characterized yeast toolkit for modular, multipart assembly. ACS Synth. Biol. 4, 975-986 (2015).

74. Kuorelahti, S., Kalkkinen, N., Penttilä, M., Londesborough, J. \& Richard, P. Identification in the mold Hypocrea jecorina of the first fungal D-galacturonic acid reductase. Biochemistry 44, 11234-11240 (2005).

75. Caron, L., Rousseau, F., Gagnon, É. \& Isenring, P. Cloning and functional characterization of a cation-Cl- cotransporter-interacting protein. J. Biol. Chem. 275, 32027-32036 (2000).

76. Bergeron, M. J. et al. Frog oocytes to unveil the structure and supramolecular organization of human transport proteins. PLoS One 6, e21901 (2011)

77. Clémençon, B., Fine, M., Schneider, P. \& Hediger, M. A. Rapid method to express and purify human membrane protein using the Xenopus oocyte system for functional and low-resolution structural analysis. In Methods in Enzymology, vol. 556, 241-265 (Elsevier, 2015).

78. Kilkenny, C., Browne, W. J., Cuthill, I. C., Emerson, M. \& Altman, D. G. Improving bioscience research reporting: the ARRIVE guidelines for reporting animal research. PLoS Biol. 8, e1000412 (2010).

79. R Core Team. R: A Language and Environment for Statistical Computing. R Foundation for Statistical Computing, Vienna, Austria (2013). 
80. Saier Jr, M. H., Tran, C. V. \& Barabote, R. D. TCDB: the transporter classification database for membrane transport protein analyses and information. Nucleic Acids Res. 34, D181-D186 (2006).

81. Madeira, F. et al. The EMBL-EBI search and sequence analysis tools APIs in 2019. Nucleic Acids Res. (2019). https://www.ebi.ac. $\mathrm{uk} /$ Tools/msa/muscle/, visited 17.12.2019.

82. Jones, D. T., Taylor, W. R. \& Thornton, J. M. The rapid generation of mutation data matrices from protein sequences. Bioinformatics 8, 275-282 (1992).

83. Kumar, S., Stecher, G., Li, M., Knyaz, C. \& Tamura, K. MEGA X: molecular evolutionary genetics analysis across computing platforms. Mol. Biol. Evol. 35,1547-1549 (2018).

84. Paradis, E. \& Schliep, K. ape 50: an environment for modern phylogenetics and evolutionary analyses in R. Bioinformatics 35 , 526-528 (2019).

85. Wickham, H. ggplot2: Elegant Graphics for Data Analysis (Springer-Verlag, New York, 2016).

\section{Acknowledgements}

This work was supported by Academy of Finland (funding decision No. 298392). Prof. Doris Rentsch of University of Bern is acknowledged for the use of her frog facility. Merja Helanterä is acknowledged for skillful technical assistance and Kari Koivuranta for providing yeast expression plasmids for STP1, STR1 and STR3, as well as GeneArt plasmids which were used for constructing CDT-1, CDT-2, Trire2_50618, Trire2_56684 (QM6a version), Trire2_72383 (QM6a version) and Trire2_79202 (QM6a version) expression plasmids. Prof. Matthias Hediger furthermore acknowledges the support of the electrophysiological functional studies of the transporters by the Swiss National Science Foundation Grant Sinergia \#CRSII5 180326.

\section{Author contributions}

S.H. designed some of the constructs, did part of the cloning, performed the experiments and analysis, and drafted the manuscript. J.P.G. performed the surgery to obtain the oocytes, participated in the electrophysiology studies, and helped in drafting the manuscript. M.V. did the manual annotations and designed some of the constructs, as well as read and commented on the manuscript. A.W.P. designed some of the constructs and did part of the cloning, as well as read and commented on the manuscript. M.H. planned the collaboration, read and commented on the manuscript. C.P.L. edited the manuscript, planned the collaboration and designed some of the studies.

\section{Competing interests}

The authors declare no competing interests.

\section{Additional information}

Supplementary Information The online version contains supplementary material available at https://doi.org/ 10.1038/s41598-021-93552-7.

Correspondence and requests for materials should be addressed to C.P.L.

Reprints and permissions information is available at www.nature.com/reprints.

Publisher's note Springer Nature remains neutral with regard to jurisdictional claims in published maps and institutional affiliations.

Open Access This article is licensed under a Creative Commons Attribution 4.0 International License, which permits use, sharing, adaptation, distribution and reproduction in any medium or format, as long as you give appropriate credit to the original author(s) and the source, provide a link to the Creative Commons licence, and indicate if changes were made. The images or other third party material in this article are included in the article's Creative Commons licence, unless indicated otherwise in a credit line to the material. If material is not included in the article's Creative Commons licence and your intended use is not permitted by statutory regulation or exceeds the permitted use, you will need to obtain permission directly from the copyright holder. To view a copy of this licence, visit http://creativecommons.org/licenses/by/4.0/.

(C) The Author(s) 2021 